\title{
Aplicação dos Métodos Evolução Diferencial e Algoritmo de Colisão de Partículas na Estimativa de Parâmetros do Modelo de Difusão Anômala
}

\author{
Luciano G. da Silva, Antônio J. da Silva Neto \\ Universidade do Estado do Rio de Janeiro, Instituto Politécnico \\ 28630-050 - Nova Friburgo, RJ \\ E-mail: luciano@iprj.uerj.br , ajsneto@iprj.uerj.br \\ Augusto C. N. R. Galeão \\ Laboratório Nacional de Computação Científica \\ 25651-076 - Petrópolis, RJ \\ E-mail: $\underline{\text { acng@lncc.br }}$ \\ Diego C. Knupp, Luiz Bevilacqua \\ Universidade Federal do Rio de Janeiro, COPPE/UFRJ \\ 21941-972 - Rio de Janeiro, RJ \\ E-mail: diegoknupp@gmail.com, bevilacqua@coc.ufrj.br
}

Resumo. Neste trabalho é realizada uma abordagem para o problema inverso do modelo de difusão anômala através dos métodos estocásticos Particle Collision Algorithm - PCA e Differential Evolution - DE, cujo objetivo é estimar os parâmetros envolvidos no modelo matemático. É apresentada uma aproximação para a solução do problema direto empregando o método de diferenças finitas, a qual é verificada com uma aproximação obtida NDSolve Routine do software Mathematica. Uma análise de sensibilidade é realizada para escolha de dados experimentais sintéticos e para um estudo de correlação entre os parâmetros.

Palavras-chaves: Difusão Anômala, Problemas Inversos, Análise de sensibilidade.

\section{INTRODUÇÃO}

A recente formulação analítica desenvolvida por Bevilacqua et al. (2011) para simular os fenômenos de difusão com retenção apresenta interessantes aplicações em diversas áreas, por exemplo em reações químicas com processos de adsorção, fluxo multifásico através de meios porosos, e espalhamento de população com retenção parcial para a garantia de domínio de território. Nesta nova formulação, desenvolvida a partir de uma abordagem discreta, considerase um parâmetro de controle que representa a fração de partículas que são capazes de se difundir e assim, regula a equação para a modelagem de difusão. Neste trabalho é apresentada uma análise de sensibilidade e a solução de um problema inverso através dos métodos estocásticos PCA e DE para a estimativa dos parâmetros envolvidos no modelo de difusão anômala.

\section{FORMULAÇÃO DO PROBLEMA DIRETO}

Bevilacqua et al. (2011), a partir de uma abordagem discreta, propuseram uma formulação matemática para o fenômeno de difusão anômala. Nesta nova abordagem, um termo diferencial de quarta ordem é introduzido, bem como um parâmetro de controle $\beta$, que representa a fração de partículas que são capazes de se difundir e assim, evita que o fenômeno de difusão ocorra isoladamente.

Um processo de difusão anômala é formulado matematicamente por

$$
\frac{\partial p}{\partial t}=\beta K_{2} \frac{\partial^{2} p}{\partial x^{2}}-\beta(1-\beta) K_{4} \frac{\partial^{4} p}{\partial x^{4}}
$$

com $0 \leq \beta \leq 1$. 
Neste trabalho são empregadas as seguintes condições de contorno

$$
\begin{gathered}
p(0, t)=1 \quad \text { para } t>0 \\
p(L, t)=1 \quad \text { para } t>0 \\
\frac{\partial p(0, t)}{\partial x}=0 \\
\frac{\partial p(L, t)}{\partial x}=0
\end{gathered}
$$

e condição inicial

$$
p(x, t)=f(x)=2 \cdot \operatorname{sen}^{100}\left(\frac{\pi \cdot x}{L}\right)+1, \text { em } 0 \leq x \leq L, \text { para } t=0
$$

\section{FORMULAÇÃO DO PROBLEMA INVERSO}

Considere que em um fenômeno de difusivo com retenção, formulado matematicamente pela Eq.(1), os valores de $\beta, K_{2}$ e $K_{4}$ sejam desconhecidos, mas que se tenha disponível alguns valores medidos experimentalmente para a variável observável $P$, neste caso, busca-se determinar os valores de $\beta, K_{2}$ e $K_{4}$ que minimizam o funcional dado pelo o somatório dos resíduos quadrados. Este é o problema inverso, considerando $\vec{Z}=\left\{\beta, K_{2}, K_{4}\right\}^{T}$ tem-se

$$
\vec{Z}=? \rightarrow \min _{\vec{Z}} Q(\vec{Z})=\sum_{j=1}^{N d}\left[P_{j}^{\text {calc }}(\vec{Z})-P_{j}^{\exp }\right]^{2}=\vec{F}^{T} \vec{F}
$$

onde $N_{d}$ é o número de dados experimentais disponíveis, $P_{j}^{\text {calc }}$ é o valor calculado da variável observável em função de $\vec{Z}, P_{j}^{\text {exp }}$ é o valor da variável observável medida experimentalmente e $\vec{F}$ é o vetor de resíduos, dado pela diferença entre o valor calculado e o valor experimental de $P$, i.e.

$$
\vec{F}=P_{j}^{\text {calc }}-P_{j}^{\text {exp }}, \text { com } j=1,2, \ldots, N_{d}
$$

\section{SOLUÇÃO DO PROBLEMA INVERSO}

O problema descrito pela Eq.(4) pode ser entendido como um problema de otimização onde busca-se determinar $\vec{Z}$ que minimize a função objetivo $Q(\vec{Z})$. Para isso, pode-se usar métodos determinísticos, estocásticos e estratégias híbridas. Neste trabalho, optou-se em utilizar os métodos estocásticos Algoritmo de Colisão de Partículas (Particle Collision Algorithm - PCA) e o Método Evolução Diferencial (Diferential Evolution - DE).

\subsection{Algoritmo de Colisão de Partículas - PCA}

O Algoritmo de Colisão de Partículas (PCA - Particle Collision Algorithm) proposto por Sacco em 2005, e desenvolvido com colaboradores [7][9], foi inspirado na interação de nêutrons com o combustível em reatores nucleares [8], basicamente em absorção e espalhamento. Se uma partícula apresenta "aptidão" ao núcleo ela é absorvida e tem seus limites explorados, caso contrário, é espalhada para outras regiões. Por apresentar essa dinâmica, o PCA pode ser caracterizado como um algoritmo de metropolis [5] e aumenta as suas chances de escapar de mínimos locais. 


\subsection{Método Evolução Diferencial (Differential Evolution - DE)}

O Evolução Diferencial (Differential Evolution - DE), proposto por Price e Storn (1995 e 1997), originou-se com o algoritmo de Recozimento Genético (Genetic Annealing - GA) desenvolvido por Kenneth Price e publicado na edição de outubro de 1994 do Dr. Dobbs Journal (DDJ). O Recozimento Genético, baseado em uma população inicial, é um algoritmo de otimização combinatória que implementa um critério de recozimento via limiares. Depois que o algoritmo de recozimento genético apareceu na DDJ, Price foi contactado pelo Dr. Rainer Storn, sobre a possibilidade de utilizar o GA para resolver o problema de ajuste polinomial de Chebyshev. Determinar os coeficientes dos polinômios de Chebyshev é considerada por muitos a ser uma tarefa difícil para um otimizador de propósito geral [6]. Desta forma, nasceu então o Evolução Diferencial.

\subsection{Análise de Sensibilidade}

A análise de sensibilidade contribui de forma significativa na formulação e solução de problemas inversos, permitindo por exemplo, a obtenção de dados experimentais com maior qualidade, i.e. que apresentam maior sensibilidade aos parâmetros que se deseja estimar.

Os coeficientes de sensibilidade são definidos como [1]

$$
\bar{X}_{Z_{l}}^{j}=\frac{\partial P_{\text {calc } j}(\vec{Z})}{\partial Z_{l}}, j=1,2, \ldots, N_{d} \text { e } l=1,2, \ldots, N_{i}
$$

onde $\vec{Z}$ é o vetor de incógnita, i.e. dos parâmetros que se deseja estimar, $N_{d}$ representa o número total de dados experimentais, e $N_{i}$ o número de incógnitas.

Neste trabalho, optou-se pelo uso dos coeficientes de sensibilidade modificados, dados por

$$
X_{Z_{l}}^{j}=Z_{l} \cdot \bar{X}_{Z_{l}=Z_{l}}^{j} \cdot \frac{\partial P_{\text {calc } j}(\vec{Z})}{\partial Z_{l}}, j=1,2, \ldots, N_{d} \text { e } l=1,2, \ldots, N_{i}
$$

Quando os coeficientes de sensibilidade apresentam valores elevados, pode-se obter boas estimativas para as incógnitas dentro de intervalos razoavelmente confiáveis, no entanto, quando duas ou mais incógnitas são estimadas simultaneamente, os coeficientes de sensibilidade podem estar correlacionados, o que afeta a grandeza observável $P_{\text {calc }}$.

\section{RESULTADOS}

\subsection{Problema Direto}

Para os resultados apresentados a seguir, foram empregadas as condições de contorno dadas pelas Eqs.(1b-e) e condição inicial dada pela Eq.(2). Foi realizada uma aproximação para a solução do problema direto através do método de diferenças finitas, bem como uma verificação desta aproximação usando o NDSolve Routine do software Mathematica.

Na Tabela 1 são apresentados os valores para os parâmetros considerados neste estudo para a solução do problema direto, outros casos testes podem ser verificados em [10].

\begin{tabular}{c}
\hline Caso Teste \\
\hline$\beta=0,2$ \\
$K_{2}=10^{-3}$ \\
$K_{4}=10^{-8}$
\end{tabular}

Tabela 1 - Valores atribuídos aos parâmetros para a solução do problema direto.

Na Figura 1, é apresentada uma comparação entre a solução numérica obtida através do método de diferenças finitas e com o NDSolve Routine do software Mathematica. 


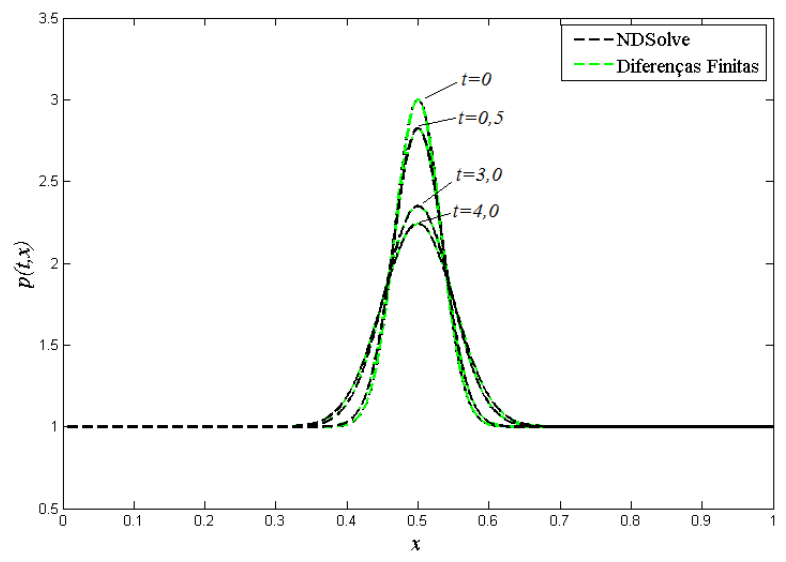

Figura 1 - Comparação entre as aproximações obtidas via diferenças finitas e NDSolve, nos tempos $t=0, t=0,5, t=3$ e $t=4$.

Observa-se na Figura 1, a boa concordância entre as soluções obtidas, via diferenças finitas e NDSolve Routine, para a equação de difusão anômala empregando os parâmetros dados na Tabela 1. Usando esta solução numérica, é possível empregar métodos determinísticos, estocásticos ou híbridos para a construção de uma rotina computacional para a solução do problema inverso e estimativa de parâmetros envolvidos no modelo de difusão anômala.

\subsection{Problema Inverso}

A solução para o problema inverso apresentada a seguir foi obtida a partir dos métodos estocásticos PCA e DE, na tentativa de estimar os parâmetros indicados na Tabela 1, i.e. $\vec{Z}_{\text {exato }}=\left\{\beta, K_{2}, K_{4}\right\}^{T}=\left\{0,2 ; 10^{-3} ; 10^{-8}\right\}^{T}$, como dados experimentais reais não estavam disponíveis, dados experimentais sintéticos foram gerados usando

$$
Y_{i}=P_{\text {calc }_{i}}\left(\vec{Z}_{\text {exato }}\right)+\sigma_{e} \cdot r, \quad i=1,2, \ldots, N_{d}
$$

onde $P_{\text {calc }_{i}}\left(\vec{Z}_{\text {exato }}\right)$ é o valor da intensidade da variável observável, $\sigma_{e}$ representa o desvio padrão, $r$ é um número aleatório gerado pelo computador no intervalo de $[0,1]$ e $N_{d}$ o número total de dados experimentais. A Eq.(4) é reescrita então como

$$
Q(\vec{Z})=\sum_{j=1}^{N d}\left[P_{j}^{\text {calc }}(\vec{Z})-Y_{j}\right]^{2}=\vec{F}^{T} \vec{F}
$$

Para os resultados apresentados nas tabelas a seguir, foram considerados um total de $N_{d}=10$ dados experimentais, adquiridos na posição $x=0,45$ para $t=1,2, \ldots, 10$. Na Figura 2, são apresentadas as curvas para os coeficientes de sensibilidade calculados a partir da Eq.(7).

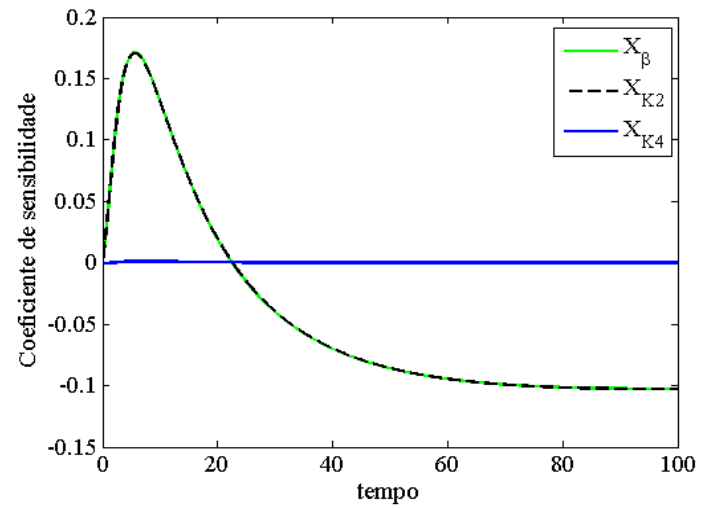

Figura 2 - Coeficientes de sensibilidade para o vetor de incógnitas $\vec{Z}=\left\{\beta, K_{2}, K_{4}\right\}^{T}$. 
Na Figura 2, nota-se a forte correlação entre os parâmetros $\beta$ e $K_{2}$, além de uma baixa sensibilidade ao parâmetro $K_{4}$. Isto indica que a possibilidade de se estimar simultaneamente estes três parâmetros é baixíssima.

\begin{tabular}{c|c|c|c|c|c|c}
\hline Método & $\beta$ & $K_{2}$ & $K_{4}$ & $\beta K_{2}$ & $\beta(1-\beta) K_{4}$ & $Q(\vec{Z})$ \\
\hline PCA & $1,0556 \times 10^{-3}$ & $1,8946 \times 10^{-1}$ & $1,5160 \times 10^{-6}$ & $1,9999 \times 10^{-4}$ & $1,5985 \times 10^{-9}$ & $6,0840 \times 10^{-11}$ \\
DE & $1,8036 \times 10^{-2}$ & $1,1109 \times 10^{-2}$ & $8,2983 \times 10^{-8}$ & $2,0035 \times 10^{-4}$ & $1,4697 \times 10^{-9}$ & $2,1324 \times 10^{-7}$ \\
$\begin{array}{c}\text { Valor } \\
\text { exato }\end{array}$ & $\boldsymbol{\beta}=\mathbf{0 , 2}$ & $\boldsymbol{K}_{\mathbf{2}}=\mathbf{1 0}^{-3}$ & $\boldsymbol{K}_{\mathbf{4}}=\mathbf{1 0}^{-8}$ & $\boldsymbol{\beta} \cdot \boldsymbol{K}_{2}=\mathbf{2} \cdot \mathbf{1 0}^{-4}$ & $\beta(\mathbf{1}-\boldsymbol{\beta}) \boldsymbol{K}_{4}=\mathbf{1 , 6} \cdot \mathbf{1 0} 0^{-9}$ & \\
\hline
\end{tabular}

Tabela 2 - Estimativa para o vetor $\vec{Z}=\left\{\beta, K_{2}, K_{4}\right\}^{T}$ empregando dados experimentais sem ruído. $\vec{Z}_{\text {exato }}=\left\{\beta, K_{2}, K_{4}\right\}^{T}=\left\{0,2 ; 10^{-3} ; 10^{-8}\right\}^{T}$.

Verifica-se na Tabela 2 que, para dados experimentais sem ruído, embora o valor da função objetivo seja próximo de zero, não foi possível obter resultados acurados para a estimativa simultânea dos três parâmetros. Porém, percebe-se que os valores dos coeficientes de cada termo diferencial, apresentam boas aproximações, i.e. $\beta K_{2}$ e $\beta(1-\beta) K_{4}$. Isto ocorre devido a forte correlação entre os parâmetros $\beta$ e $K_{2}$, e também pela baixa sensibilidade apresentada pela variável observável ao parâmetro $K_{4}$ (vide Figura 2).

\begin{tabular}{c|c|c|c|c|c|c}
\hline Método & $\beta$ & $K_{2}$ & $K_{4}$ & $\beta K_{2}$ & $\beta(1-\beta) K_{4}$ & $Q(\vec{Z})$ \\
\hline $\begin{array}{c}\text { PCA } \\
\text { DE }\end{array}$ & $9,9998 \times 10^{-2}$ & $2,2835 \times 10^{-4}$ & $3,1968 \times 10^{-4}$ & $2,2835 \times 10^{-4}$ & $6,1870 \times 10^{-9}$ & $1.0609 \times 10^{-2}$ \\
$\begin{array}{c}\text { Valor } \\
\text { exato }\end{array}$ & $\boldsymbol{\beta}=\mathbf{0 , 2}$ & $\boldsymbol{K}_{2}=\mathbf{1 0}^{-3}$ & $\boldsymbol{K}_{\mathbf{4}}=\mathbf{1 0}^{-\mathbf{8}}$ & $\boldsymbol{\beta} \cdot \boldsymbol{K}_{2}=\mathbf{2} \cdot 10^{-4}$ & $\boldsymbol{\beta}(\mathbf{1}-\boldsymbol{\beta}) \boldsymbol{K}_{\mathbf{4}}=\mathbf{1 , 6} \cdot \mathbf{1 0} 0^{-9}$ & \\
\hline
\end{tabular}

Tabela 3 - Estimativa para o vetor $\vec{Z}=\left\{\beta, K_{2}, K_{4}\right\}^{T}$ empregando dados experimentais com até $3 \%$ de ruído. $\vec{Z}_{\text {exato }}=\left\{\beta, K_{2}, K_{4}\right\}^{T}=\left\{0,2 ; 10^{-3} ; 10^{-8}\right\}^{T}$.

Acrescentando um ruído de até 3\% nos dados experimentais sintéticos, as observações feitas para dados experimentais sem ruído são verificadas, os valores dos coeficientes $\beta K_{2} \mathrm{e}$ $\beta(1-\beta) K_{4}$ não foram consideravelmente afetados. O que mostra que se forem levados em consideração apenas estes dois fatores, possivelmente, existirá uma grande chance de se estimar ambos simultaneamente.

\section{CONSIDERAÇÕES FINAIS}

Estimativa de parâmetros através de problemas inversos, em geral, requer um conjunto de estratégias para se obter bons resultados. Neste trabalho optou-se por uma análise de sensibilidade para a escolha de um conjunto de dados experimentais sintéticos na intenção de se obter boas aproximações para o conjunto de parâmetros $\left(\beta, K_{2}\right.$ e $\left.K_{4}\right)$ na equação da difusão anômala [3]. Para o problema direto, foi verificada a solução a partir de dois métodos diferentes, Diferenças Finitas e NDSolve routine do Software Wolfram Mathematica. 
Foram encontradas dificuldades para a estimativa simultânea dos três parâmetros devido a forte correlação entre $\beta$ e $K_{2}$, e também pela baixa sensibilidade para $K_{4}$ (vide Figura 3). Porém percebe-se que os valores estimados representam boas aproximações para os fatores que multiplicam, respectivamente, os termos de segunda e quarta ordem, i.e. $\beta K_{2}$ e $\beta(1-\beta) K_{4}$, o que sugere então, para trabalhos futuros, que sejam considerados apenas estes dois fatores, reduzindo para apenas dois parâmetros a serem estimados.

\section{Referências}

[1] Beck, J. V., Blackwell, B. and St. Clair Jr., C. R., Inverse Heat Conduction - Ill-Posed Problems, Wiley-Interscience, 1985.

[2] Bevilacqua, L., Galeão, A. C. N. R. and Costa, F. P., A new analytical formulation of retention effects on particle diffusion process, Annals of the Brazilian Academy of Sciences, Vol. 83, No. 4, pp. 1443-1464, 2011.

[3] Bevilacqua, L., Galeão, A. C. N. R. and Costa, F. P., On the Significance of Higher Order Differential Terms in Diffusion Processes. Journal of the Brazilian Society of Mechanical Sciences and Engineering, v. 34, p. 166-175, 2011a.

[4] D.W. Marquardt, An Algorithm for Least-Squares Estimation of Nonlinear Parameters, J. Soc. Industr. Appl. Math., Vol. 11, pp. 431-441, 1963.

[5] Metropolis, N.; Rosenbluth, A. W.; Rosenbluth, M. N.; Teller, A. H.; Teller, E.. Equation of state calculations by fast computing machines, Journal of Chemical Physics, v .21, pp.1087$1092,1953$.

[6] Price, K.; Storn, R.; Lampinem, J. A. Differential Evolution : A Practical Approach to Global Optimization. Berlin Heidelberg: Springer-Verlag, 2006.

[7] Sacco, W. F.; Oliveira, C. R. E. A new stochastic optimization algorithm based on particle collisions, 2005 ANS Annual Meetings, Transactions of the American Nuclear Society, San Diego, EUA, 2005.

[8] Sacco, W. F.; Knupp, D. C.; Luz, E. F. P.; Silva Neto, A. J. Algoritmo de Colisão de Partículas (Particle Collision Algorithm), em Técnicas de Inteligência Computacional Inspiradas na Natureza - Aplicações em Problemas Inversos em Transferência Radiativa (Ed.), SBMAC, São Carlos, Capítulo 9, pp. 79-89, 2009.

[9] Sacco, W. F.; Oliveira, C. R. E.; Pereira, C. M. N. A. Two sthocastic optimization algorithms applied to nuclear reactor core design, Progress in Nuclear Energy, v. 48, pp. 525539, 2006.

[10] Silva, L. G. Problemas inversos em processos difusivos com retenção. 2013. 105 f. Dissertação (Mestrado em Modelagem Computacional) - Instituto Politécnico do Rio de Janeiro, Universidade do Estado do Rio de Janeiro, Nova Friburgo, 2013. 